\title{
CONFINED WETting OF FOCA CLAY POWDER/PELLET MIXTURES: EXPERIMENTATION AND NUMERICAL MODELING \\ Pascal Maugis ${ }^{a, 1}$, Christophe Imbert $^{b}$ \\ ${ }^{a}$ Commissariat à l'Énergie Atomique, DMT/MTMS, Saclay, 91191 Gif-sur-Yvette
} Cedex, France. Now at LSCE, Orme des Merisiers, 91191 Gif-sur-Yvette Cedex, France.

${ }^{b}$ Commissariat à l'Énergie Atomique, DPC/SCCME, Saclay, 91191 Gif-sur-Yvette Cedex, France.

\begin{abstract}
Potential geological nuclear waste disposals must be properly sealed to prevent contamination of the biosphere by radionuclides. In the framework of the RESEAL project, the performance of a bentonite shaft seal is currently studied at Mol (Belgium). This paper focuses on the hydro-mechanical physical behavior of centimetric, unsaturated samples of the back-filling material - a mixture of FoCa-clay powder and pellets - during oedometer tests. The hydro-mechanical response of the samples is observed experimentally, and then compared to numerical simulations performed by our Cast3M Finite Element code. The generalized Darcy's law and the Barcelona Basic Model mechanical model formed the physical basis of the numerical model and the interpretation. They are widely used in engineered barriers modeling.

Vertical swelling pressure and water intake were measured throughout the test. Although water income presents a monotonous increase, the swelling pressure evolution is marked by a peak, and then a local minimum before increasing again to an asymptotic value. This unexpected behavior is explained by yielding rather than by heterogeneity. It is satisfactorily reproduced by the model after parameter calibration.

Several samples with different heights ranging from 5 to $12 \mathrm{~cm}$ show the same hydro-mechanical response, apart from a dilatation of the time scale. The interest of the characterization of centimetric samples to predicting the efficiency of a metric sealing is discussed.
\end{abstract}

keywords: hydro-mechanics, clay, heterogeneous media, swelling pressure, plasticity

\section{Résumé}

La capacité de scellement d'un puits d'accès à un stockage de déchets radioactifs par un bouchon de bentonite est en cours détude à Mol (Belgique). Ce papier aborde le comportement hydro-mécanique d'échantillons centimétriques non saturés du matériau de remplissage, constitué par un mélange de poudre et de berlingots d'argile FoCa. Chaque échantillon est confiné dans un oedomètre, mouillé par la

\footnotetext{
${ }^{1}$ Corresponding author. Fax: +33 1690877 16. Email-address: pmaugis@cea.fr (P. Maugis).
} 
base, et exposé à l'air ambiant en son sommet. Le présent travail tente de comprendre et de reproduire la réponse hydro-mécanique des échantillons, à l'aide de la loi de Darcy généralisée et du modèle de base de Barcelone, implémentés dans notre code aux Eléments Finis Cast3M .

L'entrée d'eau montre une imbibition progressive et asymptotique, alors que la pression de gonflement présente une variation non monotone avant de tendre vers une valeur asymptotique. Ce processus s'explique par plastification plutôt que par l'hétérogénéité, et est bien reproduit par le modèle calibré.

Les échantillons, de 5 à $12 \mathrm{~cm}$ de hauteur, montrent la même réponse hydro-mécanique, suggérant ainsi une invariance d'échelle précoce compte tenu de la taille des berlingots. L'utilité de cette caractérisation vis-à-vis de la prédiction de l'efficacité d'un scellement métrique est discutée.

Mots-clefs : hydro-mécanique, argile, milieu hétérogène, pression de gonflement, plasticité

\section{Introduction}

Geological disposal of high-level radioactive waste requires long-term insulation from biosphere. This is a pre-requisite to acceptance by the public of waste repository as a safe solution to end-of-life nuclear fuel (AEN 1999). The backfilling and sealing of shafts and galleries are essential steps in underground repository designs. All openings created during the repository construction would constitute potential preferential pathways for migration of water, gas and radionuclides (de Marsily 2002). They need to be effectively sealed afterward. The RESEAL (for REpository SEALing) project aims at demonstrating that proper sealing of boreholes and shafts can be achieved using bentonite. This project includes: selection of the sealing material; development of the seal installation technique, borehole and shaft sealing tests monitoring, and modeling of the main processes observed during the tests. These demonstration tests are performed in the HADES Underground Research Facility (Mol, Belgium) (Volckaert et al. 1996a). This work follows a number of previous works on various experimental and modeling efforts in the frame of RESEAL Project (Le Potier et al. 1998 ; Imbert et al. 2002), and other similar projects like CATSIUS CLAY (Volckaert et al. 1996a), BACCHUS 2 (Volckaert et al. 1996b), PRACLAY (de Bruyn and Boyazis 1996), INTERCLAY (Jeffries 1992) or ANDRA conducted projects (Robinet 
et al. 1995 ; Robinet et al. 2002).

FoCa clay is a kind of bentonite. From its swelling properties depends its efficient embedding. The ability to swell under imbibition is closely related to the material dry density. The dry density of sole FoCa powder is too low to yield consequent swelling potential. On the other hand, compacted blocks are very dense and yield very high swelling pressure. They do not allow high precision filling of gaps and holes however and leave open pathways to interstitial flows. Moreover, cohesion between blocks is poor. Mixtures composed of bentonite powder and high dry density bentonite pellets have both advantages (i) to properly fill-in cavities of any shape, and (ii) to yield substantial imbibition swelling pressure necessary to maintain good quality closure for a long time. The mixture is also rather cheap to produce and offers good overall cohesion. Compaction efforts can thus be alleviated by their use instead of pre-compacted blocks. That is why powder/pellet mixtures, produced in an industrial way, should allow a substantial reduction of the waste repository installation cost, as well as strengthened efficiency.

Performance assessment requires this material to be characterized in order to understand its effectiveness as a seal. FoCa powder has already been under focus, and characterized (Volckaert et al. 1996a). Powder/pellet mixtures are, however, expected to behave somewhat differently, both from hydraulic and mechanical points of view. It is yet not sure whether this heterogeneous medium follows the same laws as sole powder. Even if it proves so, the parameters of these laws are expected to be significantly different from that of powder.

Small scale lab experiments are run as means to identify basic physical processes. Large scale experiments undergo natural and technical solicitations directly related to industrial sealing performances. Whether and how the knowledge acquired on small scale samples can be useful for the performance assessment of large scale seals are questions which arise naturally. In order to answer this question, the response of the material must be investigated with samples of increasing heights, across several orders of magnitude. This study focuses on small-scale, centimetric samples, as part of the characterization process. Let us emphasize, however, the difficulty to address the question of changes in material properties with developing scales because: (i) decimetric samples require very long monitoring scales (several years), and (ii) as a consequence of this, large scale experiments - like the metric plug experimented in Mol, but also decimetric tests - must be conducted under different experimental protocols in order to accelerate the processes, 
like pressurized water injection around or inside the medium. (iii) in large scale in situ tests, the behavior of the geological host is unknown. This makes the determination of the properties of the clay buffer more difficult. From this point of view, the range of our study is limited to observation and understanding of small scale samples, as a qualitative way to understand the response of higher scale systems.

Oedometer tests have been conducted on several samples of different heights in order to understand how the material properties may vary within one length scale. This methodology addresses the significance of the characterization that might be deduced from specimens that are small with respect to their internal heterogeneity scale. The heights of the samples were limited by the necessity to have reasonable monitoring times. They did not exceed 12 $\mathrm{cm}$, for which full hydration already needs one year. These experiments and their results are described in the first section of this paper. They continue previous works (Villar and Martin 1996 ; Pasquiou 2001 ; Imbert et al. 2002).

The modeling of the oedometer tests has been conducted and is described in the second part. Hydraulics were modeled by the classical generalized Darcy's law for unsaturated media. The reasons for this choice are its simplicity, and its relative independence from the mathematical forms of permeability and capillary laws. Indeed, in most cases, parameters can be inferred to fit experimental observations. Porosity has been assumed totally circulating, i.e. cinematic porosity is equal to total porosity. Disregarding the minor amount of water involved in crystalline and interlamellar spaces, this assumption consists mainly in allowing water adsorbed in the double-layers to flow like capillary water (i.e. governed by a 'matric' potential) (Yong 1999 ; Jullien et al. 2005). According to Achari et al. (1999), the fraction of adsorbed water thus assimilated to free water would be exaggerated by the use of deionized water. Yong (op. cit.) insists on the importance of a differentiated modeling of matric potential- and osmotic-driven flows. However, this discrimination appears necessary insofar as the mechanical model requires so. It would produce little enhancement to the hydraulic modeling specifically.

Concerning the mechanical behavior of bentonites, a number of models is available in the literature. Barnel (2004) considered the Barcelona Basic Model (BBM) appropriate, because of its phenomenological approach that enables it to fit most of the experimental protocols conducted on swelling clays (Alonso et al. 1990 ; Gens and Alonso 1992 ; Volckaert et al. 2000). It does not meet thermodynamical requirements however and is not based on elasto-plasticity theory. In contrast, thermodynamical models (Coussy 
2004) have the concurrent advantage to satisfy thermodynamical equilibrium. Other models are based on micromechanics and microstructure (Chateau and Dormieux 1998 ; Dormieux et al. 2003). Following this line, Alonso et al. (1999) further developed the BBM model by coupling macro- and micro-structure, and by introducing Suction Decrease plastification in order to be able to reproduce particular behavior of highly active clays. Microscopic expansion may result in macropore reductions. Therefore, the repartition of swelling across the different scales (crystalline, inter-layer, aggregatesplitting, inter-aggregate, inter-granular water) is subject to the different corresponding imbibition processes which might be as various as solvation, osmosis, diffusion of adsorbed water or suction (Saiyouri et al. 2004 ; Jullien et al. 2005 ; Suzuki 2005). BBM does not differentiate these kinds of porosity, and considers solely suction yielding during drying. BBM explicitly excludes crystalline as well as osmotic swelling, yet expected in bentonite (op. cit.). Thus, great care must be taken as to conclude on real internal processes in the light of this model. It will be reminded in the interpretation of the tests. This should be the object of further modeling studies. Nevertheless, our simulations would give a possible explanation of the results of oedometer tests, under the assumption of irreversible strains induced by increasing suction paths or increasing stress paths only.

Permeability variations due to deformations of the material were accounted for by isotropic Kozeny-Carman model, based on porosity (Kozeny 1927 ; Carman 1937). The choice of a unique scalar parameter like porosity has been questioned in the case of important shear deformations, where anisotropic changes in permeability should occur (Wong 2003). Given the anisotropic compression, generated by the anisotropy of stresses which is to be expected between radially confined and longitudinally impregnated samples, it is presumable that permeability should also become anisotropic under this solicitation. Although it may be of importance in multi-dimensional wetting of a sealing buffer, the $1 \mathrm{D}$ oedometer test should not be sensible to the discrimination of vertical and horizontal permeability. A reason for this is that vertical permeability takes the largest part to the hydration kinetics. However, this very fact is another difficulty in considering model data, gained from oedometer tests, as meaningful in forecasting the behavior of three-dimensional sealing applications. Other limitations of the KozenyCarman law reside in the existence of immobile water (Chapuis and Aubertin 2003), or in less likely pore-connectivity closure by precipitation (Ontoy et al. 2003). Other authors nevertheless use Kozeny-Carman law with satisfactory results in illite and Na-bentonite with low hydraulic gradients (Dixon et al. 1999), sandstone/shale mixtures (Crawford et al. 2002) and kaolinite (Dudoignon et al. 2004). It appears from this review that, despite some 
uncertainty resulting from secondary porosity, Kozeny-Carman relationship should provide satisfactory orders of magnitude of hydraulic permeability in our tests.

Previous characterizations on sole FoCa powder gave initial parameter values for the model (Volckaert et al. 1996a ; Volckaert et al. 2000). It is expected from the numerical simulations to derive indicative orders of magnitude to parameter values, as well as plausible description of inner processes.

\section{Description and results of the oedometer tests}

\subsection{Principle}

The device is composed of an oedometric cell made of stainless steel, $120 \mathrm{~mm}$ in internal diameter, as illustrated in Fig. 1. Each carefully handmade clay mixture is placed inside its cell by alternate layers of equally weighted powder and pellets, aiming at obtaining an homogeneous repartition. They are sandwiched between stainless steel filters. The upper filter allows fluid exchanges (air and liquid water) with ambient controlled atmosphere, while the lower one permits water input via draining pipes. This three-piece ensemble is then inserted into a rigid cylinder between two pistons. Finally, this unit is placed into a rigid frame equipped with a dynamometer and a vertical displacement sensor. The former will provide the total axial swelling load opposed by the clay to the upper piston, and the latter will give an a posteriori evidence whether proper volumetric confinement has been achieved. The cell can receive samples from $3 \mathrm{~cm}$ up to 15 $\mathrm{cm}$ high. The sample preparation is carried on by compaction. To obtain the targeted density, slow uni-axial vertical compression is exerted until apparent density slightly exceeds it. In such a manner, the release of residual elastic deformations during load retrieval, stabilizes the sample to the desired dry density. This pre-compaction requires iterations and multi-step loading.

The pressure necessary to reach $1.6 \mathrm{~g} / \mathrm{cm}^{3}$ dry density is close to $8 \mathrm{MPa}$. Three samples were prepared in such a way, with final heights 5, 10 and 12 cm respectively.

[ Figure 1 about here ] 
Next step in the experiment is hydration. At $t=0$, The lower pipe is connected to a water column one meter high (0.01 MPa approximately). The level is ensured by frequent and measured filling. In the $12 \mathrm{~cm}$-high test, a high-pressure water pump is used instead, in order to accelerate hydration by imposing 6 Bar water pressure. The upper pipe allows air and vapor outflow to the temperature-controlled, dessicated atmosphere.

The water used for imbibition has been distilled and de-aired beforehand to ensure the stability of the chemical composition of water. The test therefore remains neutral from the chemical point of view. The dependence of clay properties on water geochemical composition is well know. Therefore, a consistent uncertainty should be introduced by use of natural water. The drawback of distilled water lies in its absence of ions, which artificially intensifies osmosis, perhaps beyond the realistic range. It has been specifically observed by Villar (2006), who compared in granite/bentonite mixtures the water intake kinetics between deionized water and saline water: the former flows in earlier than the latter, but more slowly in later stages. Mineral water could have been used instead in our experiments but: (i) their composition are not totally stable along time and vary between samples; (ii) the choice of a particular site-specific water results in the same problem of transposability of the observations to an other kind of water. An alternative to distilled water could be the equilibrium water of FoCa material, which would be somewhat reproducible and would also minimize osmosis. Such a proposition remains for upcoming experimentations. Use of distilled water is expected to decrease hydraulic permeability, by porosity reduction due to swelling of the double layer quasi-crystal (Suzuki 2005).

The volume of water absorbed by the sample is recorded throughout impregnation, with an estimated error around $6 \%$. The amount of vapor leaving the sample by the upper pipe has not been measured and may introduce a small bias in the water balance.

All conditions lead to a 1D hydration experiment, confirmed by a posteriori destructive measurements of water content and bulk density. The axial force $F_{z}$ exerted by the clay on the piston, divided by the piston area $S$, gives the vertical stress $\sigma_{z z}$ imposed by the piston. This stress will be called "swelling pressure", $P_{g}$, throughout this paper:

$$
\text { at a point } z=z_{\text {piston }}, \quad P_{g}=\sigma_{z z}=\frac{F_{z}}{S}
$$




\section{$2.2 \quad$ Results}

We present here two physical observations only: the total amount of liquid water that entered in the sample by its lower base during the experiment; and the "swelling pressure" $P_{g}$ defined above. The piston displacement did not exceed $0.5 \%$ of sample height. Thus, overall volume will be considered constant throughout the experiment.

The hydraulic process should theoretically be very simple: water penetrates monotonously into the sample, until reaching full imbibition; then, the in-flow rate should be constant, equal to permanent saturated Darcy flow plus evaporation plus leakage. This rate has been estimated to 1.6, 8.8 and $8.6 \mu \mathrm{l} / \mathrm{h}$ for each 12,10 and 5 -cm high samples respectively. Given the mean head gradient and the order of magnitude of intrinsic permeability, it is straightforward to calculate that permanent Darcy flow is much lower than the measured inflow. The bigger sample being supplied in water by a pump with no possible evaporation from there, total evaporation from top evacuation plus leakage amounts to $1.6 \mu l / h$. Evaporation from the feeding burette can then be roughly evaluated to $7 \mu l / h$. Both values seem realistic. Leakage and evaporation, assumed constant all along the hydration, have been filtered out to obtain the estimated water intake (Fig. 2a).

The initial pore volume is featured by the horizontal dashed line in Fig. 2a. Overall pore volume does not seem to be conservative, in accordance with standard mechanical model. One must however consider two experimental artifacts that could modulate the importance of the reorganization of pore geometry: (i) overestimation of initial water content resulting from differences in equilibrium moisture content of the powder between the thermal desiccator and the controlled air; and (ii) parietal leakage. We tried to alleviate the first one by leaving the samples long enough in ambient air, before use, in order for them to equilibrate with atmosphere. The second possibility conjectures preferential pathways along the wall of the confining cylinder, despite the strong initial compaction. In any case, this macroporosity should occlude very early as soon as swelling occurs. All in all, the measured water intake should be reliable.

\section{[ Figure 2 about here ]}

Swelling pressure measurements are presented Fig. 2b. In contrast with water intake monotony, swelling pressure yields a particular evolution: It first raises to a local maximum, then goes to a local minimum and increases again 
at about the same rate before reaching an asymptotic value. The observation of this "saddle"-like shape has been confusing. Experimental protocol and device have been first incriminated, but revealed finally correct. Heterogeneity of the material has then been considered, but same experiments, conducted later on powder only, gave the same type of variation (Fig. 3, with lower values though, because the sample had a lower dry density). The explanation must be sought elsewhere and is proposed in section 3 .

\section{[ Figure 3 about here ]}

Intensity and kinetics of the swelling are strikingly similar for samples of differing heights. They differ only in their time scale. Therefore, mechanical model and parameters seem size-independent at the 1D, centimetric scale. From the stochastic point of view, this behavior means that samples as small as $5 \mathrm{~cm}$ high and $10 \mathrm{~cm}$ in diameter are large enough to represent their class of size. In other word, the asymptotic height beyond which the material characteristics become independent of its size is less than $5 \mathrm{~cm}$. This value is surprisingly small in comparison with the internal characteristic length of heterogeneity, which is around $2 \mathrm{~cm}$.

The relatively small discrepancies observed for the different samples may partly be explained by several differences in the experimental protocol: (i) the three samples differ in the imposed average hydraulic gradients, with respective values 20,10 and $4.2 \cdot 10^{6}$ for the 5,10 and $12 \mathrm{~cm}$-high samples respectively. This is expected to have an impact in our context of non-linear unsaturated behavior; (ii) the bigger sample yields a high density $1.61 \mathrm{~g} / \mathrm{cm}^{3}$ instead of 1.6; and finally, (iii) the powder constituting part of the first sample was about twice as humid initially as the powder of the other two samples (water weight ratio of the powder equaled $11.83 \%$, instead of $5.07 \%$ ).

\section{Examination of the physical processes}

The powder/pellet mixture is obviously a heterogeneous medium. Even though their visual inspection at full saturation shows apparent homogeneity, this issue should be remembered during the interpretation. At the centimeter scale, it is expected that this heterogeneity would impact the hydromechanical response. We will use an homogeneous description of the samples, and try to mimic their behavior with a unique set of parameters. It must be emphasized that the purpose of this work is to show that it is possible to describe the overall behavior of a clay mixture at the laboratory scale. 
We leave to other studies the inference of the lower-scale processes, as long as it is not necessary to do so. Moreover, the previous section strengthens the assumption of a hydro-mechanical behavior "as if" the medium was homogeneous. This hydro-mechanical up-scaling endeavor was encouraged by previous up-scaling analysis (Genty et al. 1999 ; Volckaert et al. 2000), which showed that hydraulics of powder/pellet mixtures could be satisfactorily reproduced by use of up-scaled permeability laws.

The BBM model is built on three variables: (i) water suction $s$ (deficit of water pressure below air pressure, positive in vadose zone), which must be understood rather as 'matric potential', and is assumed to encompass and resume all other imbibition processes like osmosis; (ii) isostatic net pressure $p^{\prime}$ (excess of total stress above air pressure); and (iii) confinement stress $q$. The plastic limit is a 3D-envelop in the $\left(p^{\prime}, q, s\right)$ space. Elastic and plastic deformations are related to the variations of those three variables. Elastic deformations come independently from suction and stress. Plastic deformations induce an equivalent hardening of both Suction Increase (SI) and Loading/Collapse (LC) plastic limit curves. A simple linear elastic model (Scheidegger 1960) generalizes the BBM model to non-uniaxial problems. A further modification redirects the use of net stress to effective stress (excess of total stress above water pressure) in saturated conditions (see Appendix B for details).

The computations have been conducted with the purpose of describing spatial and temporal evolution of inner variables like elastic and plastic deformations, axial, isostatic and deviatoric stresses and strains, as well as consolidation pressure. From this post-processing and in the restrictive framework of the BBM model, it is possible to describe how a swelling clay sample can responds to wetting in such a $1 \mathrm{D}$ experiment.

\subsection{Swelling pressure first decrease}

This section details the internal hydro-mechanical response of a sample, treated as homogeneous. It gives an explanation on how hydration, by producing swelling, initiates combined actions of stress and suction, which causes different types of deformation. The occurrence of yielding by radial confinement and its consequence on the vertical stress are shown. The $5 \mathrm{~cm}$-high sample is under focus, for the purpose of the analytical description that follows.

Mechanical transformations can be assumed quasi-static, since mechani- 
cal equilibrium is a matter of seconds, whereas wetting kinetics has a magnitude of hours to months. It follows that vertical stress $\sigma_{z z}$ and radial stress $\sigma_{r r}$ remain both constant along the vertical and radial axis respectively. Therefore, spatially constant $\sigma_{z z}(t)$, noted $\sigma_{z}$, and $\sigma_{r r}(z, t)$, noted $\sigma_{r}$ will be considered. Non-diagonal stress terms, however, do vary spatially, but they are not involved in our interpretation.

Total radial deformation $\varepsilon_{r}^{t o t}$ is the sum of elastic (superscript $e l$ ) and plastic (superscript $p l$ ) contributions, which are themselves the sum of separated "net stress" (superscript $\sigma$ ) and suction (superscript $s$ ) effects (see Appendix B for details):

$$
\varepsilon_{r}^{t o t}=\varepsilon_{r}^{e l, s}+\varepsilon_{r}^{p l, s}+\varepsilon_{r}^{e l, \sigma}+\varepsilon_{r}^{p l, \sigma}
$$

To understand how yielding affects the mechanical behavior of the material, let us consider a purely elastic state (noted with superscript no plast.) modified by plasticity contribution (noted with prefix $\delta$ ):

$$
\varepsilon=\varepsilon^{\text {noplast. }}+\delta \varepsilon
$$

Yielding occurs when the couple ( $\left.\sigma^{\text {noplast. }}, s^{\text {noplast. }}\right)$ does not belong to the elastic domain. In our model, yielding under suction decrease is not taken into account. Since, in our hydration case, suction can only decrease, we can then write:

$$
\overline{\bar{\varepsilon}}^{p l, s}=0
$$

Where imbibition occurs (at the bottom), radial suction-induced elastic deformation $\varepsilon_{r}^{e l, s}$ increases by swelling, but confinement imposes

$$
\varepsilon_{r}^{t o t}=0
$$

from eq. (3.1), we get the useful relationship:

$$
\varepsilon_{r}^{e l, \sigma}+\varepsilon_{r}^{p l, \sigma}=-\varepsilon_{r}^{e l, s}
$$

How does yielding take place ? The idea is that imbibition swelling generates positive radial elastic deformation $\varepsilon_{r}^{e l, s}$ at start, eq. (B.13). $\varepsilon_{z}^{e l, s}$ is also generated with a lesser magnitude. Radial confinement imposes (eq. (3.5)) an equal negative radial elastic strain $\varepsilon_{r}^{e l, \sigma}$, corresponding to an equilibrated increase in isostatic pressure $p^{\prime}$ (eq. (B.12)).

If isostatic pressure $p^{\prime}$ remained below consolidation pressure $P_{0}, \varepsilon_{r}^{p l, \sigma}$ would remain null, and radial compressive stress would increase. In our particular case, the plastic limit $P_{0}$ is initially low - about 2.3 $\mathrm{MPa}$ in our 
example - whereas $p^{\prime}$ raises quickly above this value, leading to an immediate excess of $p^{\prime}$ before $P_{0}$, as shown in Fig. $4 \mathrm{a}$ and $4 \mathrm{~b}$. Mechanical variables thus yield discrepancies $(\delta)$ from their values without yielding. Here, radial compressive (negative) plastification $\delta \varepsilon_{r}^{p l, \sigma}$ takes place, as shown in Fig. 4c. This yielding process will be maintained afterward by $P_{0}$ collapse (Fig. $4 \mathrm{~b}$ ) under saturation (eq. B.9).

\section{[ Figure 4 and 5 about here ]}

Hence, radial yielding occurs with the same kinetics as the imbibition front. As an illustration of this, the comparison of Fig. 5a and 5b shows that the height at which radial yielding appears corresponds to a constant saturation of about $90 \%$.

We will now explain the consequence of this yielding on vertical stress. From eq. (3.1) and (3.4), we can write the relationship between plastic deformations variations:

$$
\delta \varepsilon_{r}^{e l, s}+\delta \varepsilon_{r}^{p l, s}+\delta \varepsilon_{r}^{e l, \sigma}+\delta \varepsilon_{r}^{p l, \sigma}=0
$$

Equation (3.3) stipulates that the second term is null. Moreover, the elastic deformation under suction $\varepsilon_{r}^{e l, s}$ depends only on the hydration kinetics (eq. (B.13) and (B.15)). It is only marginally influenced by mechanics. $\varepsilon_{r}^{e l, s}$ depends therefore very weakly on yielding, so that the first term of (3.6) can also be neglected. We then get:

$$
\delta \varepsilon_{r}^{e l, \sigma}=-\delta \varepsilon_{r}^{p l, \sigma}
$$

A similar result can be obtained with vertical deformations $\varepsilon_{z}$ :

$$
\delta \varepsilon_{z}^{e l, \sigma}=-\delta \varepsilon_{z}^{p l, \sigma}
$$

Because the plastic potential is a function of the square of isostatic pressure (eq. (B.16)), the plastic deformations $\delta \varepsilon_{z}^{p l, \sigma}$ and $\delta \varepsilon_{r}^{p l, \sigma}$, which are its derivative relative to $\sigma_{z}$ and $\sigma_{r}$ respectively, have common terms of same sign in both $\sigma_{z}$ and $\sigma_{r}$. Although different, $\delta \varepsilon_{z}^{p l, \sigma}$ and $\delta \varepsilon_{r}^{p l, \sigma}$ are therefore co-induced during yielding. In other words, the generation of positive $\delta \varepsilon_{r}^{p l, \sigma}$ comes in pair with a concomitant positive $\delta \varepsilon_{z}^{p l, \sigma}$. It follows, from eq. (3.8), that $\varepsilon_{z}^{e l, \sigma}$ decreases. This happens because, in the classical linear elasticity expression, expressed in cylindrical transformations:

$$
\delta \sigma_{z}=\frac{E}{1+\nu}\left(\frac{2 \nu}{1-2 \nu} \delta \varepsilon_{r}^{e l ., \sigma}+\frac{1-\nu}{1-2 \nu} \delta \varepsilon_{z}^{e l ., \sigma}\right)
$$

both right-hand terms are negative. This value $\delta \sigma_{z}$ is the correction to apply to $\sigma_{z}$ as a consequence of yielding. 


\subsection{Second increase to asymptotic state}

Variation of consolidation pressure $P_{0}$ results from two competitive processes. On the first hand, $P_{0}$ reduces to $P_{0}^{*}$ as water content increases (eq. (B.9)); and, on the other hand, $P_{0}^{*}$ is raised by plastification (eq. (B.18)). This effect, phenomenologically speaking, corresponds to high inclination of wet material to stress-induced plasticity, whereas dry material are less affected. In the end, $P_{0}$, after a first decrease by dominant imbibition, gets higher again by the newly dominant consolidation, as shown in Fig. 4b. This consolidation, in turn, slows down yielding, thus allowing vertical stress to increase again under swelling pressure solicitation. This, by the way, is why a monotonous increase of $\sigma_{z z}$, may be observed on highly over-consolidated samples in oedometer-like experiments.

Asymptotic state is achieved when the whole sample is saturated.

\section{Parameter estimation}

We focus, in this part, on the ability of the model to reproduce the response of the mixture during oedometer tests. The parameter estimation and the evaluation of its level of confidence are also addressed. The shape, amplitude and relative kinetics of the water intake and of the swelling pressure seem independent of the sample height, apart from a timescale parameter $t_{1}$ equal to the time to reach the local maximum (Fig. 2). We will therefore make an important assumption that all the samples yield the same behavior, along with the same parameter values; and that the time scales of the three samples depend only on their height rather than on differences in the value of the parameters.

In the perspective of the sealing of nuclear waste disposal, which must prove very long-term efficiency, the transitory swelling phase is of lesser interest than the asymptotic swelling pressure. In this frame, the above assumption is acceptable. As a consequence, the parameters deduced from one experiment among the three, would equally describe any of them, except maybe for the time scale.

The parametric characterization has been conducted on the smaller sample ( $5 \mathrm{~cm}$ high). In a first step, the optimal set of parameters must be chosen. Parameter calibration can then take place. 


\subsection{Sensitivity analysis}

The parameter estimation has been conducted on the basis of two experimental data: temporal variation of swelling overall pressure at the top of the sample, and water intake. Given the many parameters required by hydraulic and mechanical models, these two curves reveal immediately insufficient for full estimation. The equifinality of parameter estimation is very strong, as a number of different protocols should be used to determine separately and with correct discrimination the value of all parameters. The sole oedometer test fails to discriminate many pairs of parameters. A reduced set of them must be chosen. As a consequence of this somewhat arbitrary process, their adjusted values should be considered exclusively for their ability in reproducing the output curves, but not in their representing the real material properties.

The efficient information used as a criterion for good match between computed and experimental results was observed to be the following:

- hydraulic objectives (water intake): initial slope, asymptotic value, time to "reach" asymptote

- mechanical objectives (swelling pressure): initial slope, secondary slope, first maximum height, time to reach first maximum, saddle "depth", slope of the second increase, asymptotic value.

With this observation set, no more than three hydraulic parameters and seven mechanical parameters can be identified. The focus has been placed on parameters whose influence were direct, intense, and easily physically understandable. Given these constrains and priority, we chose the following set of eight parameters: cinematic porosity (assumed equal to total porosity) $\omega$; intrinsic permeability $k_{\text {int }}^{0}$; initial consolidation pressure $P_{0}$; over-consolidated stiffness to net mean stress $\kappa_{0}$ and $\alpha_{\kappa}$; over-consolidated stiffness to suction $\kappa_{s 0}$ and $\alpha_{\kappa s}$; stiffness to consolidation under net mean stress increase $\lambda_{0}$; with the precision that redundant Kozeny porosity $\omega_{k}$ has been set to $\omega$.

Hydraulics revealed essentially independent of the mechanical parameters, and were parameterized first. This process proved easy because of the straight-forward influence of porosity and intrinsic permeability: $\omega$, taken as total available pore volume per unit volume of material, controls asymptotic water intake; and $k_{i n t}^{0}$ controls imbibition kinetics.

Mechanical parameters were harder to determine because of their intricate influences. Among all these, let us point out the crucial importance 
of consolidation pressure $P_{0}$, the value of which may affect the qualitative response of the material. When initial $P_{0}$ is low, plasticity comes into place early, generating a deep decrease as well as a low asymptotic value of swelling pressure. The local minimum of swelling pressure $P_{g}$ may completely fade, in certain cases, as initial consolidation $P_{0}$ gets high enough, yielding very high final swelling pressure (see Fig. 6 for an example on an synthetic sample). The preparation of the samples has a fundamental influence on the initial value of $P_{0}$. They are compacted initially at about $8 M P a$, but the very dense pellets themselves (about $1.9 \mathrm{~g} / \mathrm{cm}^{3}$ ) endure up to $40 \mathrm{MPa}$ compression during their molding. In contrast, powder pre-consolidation is low but undetermined. This leads to a large uncertainty in initial $P_{0}$, as opposed to its primary importance in the overall behavior of the material.

\section{[ Figure 6 about here ]}

\subsection{Calibration results}

The main difficulty in this parameter estimation is the non-uniqueness of the acceptable set of values. Equally satisfactory results can be obtained with significantly different parameter values (up to $30 \%$, see Fig. 7). This gives an idea of the uncertainty of the characterization. On the other hand, any set of parameters that respects the experimental results is valid, just because of its ability to reproduce reality. This requires, unfortunately, to give up part of the physical understanding of parameters variations. The use of such values must also be limited to similar experimental protocols.

The $5 \mathrm{~cm}$-high sample had the following initial state: pellets dry density $1.894 \mathrm{~g} / \mathrm{cm}^{3}$ and mass water fraction $4.31 \%$, powder mass water fraction $11.83 \%$. One of the best fits, presented in Fig. 7, has been obtained with the following parameter values: $k_{i n t}^{0}=3.375 \cdot 10^{-21} \mathrm{~m}^{2} ; \omega=0.455 ; \kappa_{s 0}=0.45$; $\alpha_{\kappa s}=0.011 ; \kappa_{0}=0.015 ; \alpha_{\kappa}=0.12 ; P_{0}=2.3 M P a ; \lambda_{0}=0.22$.

These values are reasonably close to other estimations (Volckaert et al. 2000 ; Imbert et al. 2002).

\section{[ Figure 7 about here ]}

Porosity, however, is above the theoretical value deduced on density basis:

$$
1-\frac{\rho_{\text {dry }}}{\rho_{\text {solidgrains }}}
$$

With $\rho_{d r y}=1.6 \mathrm{~g} / \mathrm{cm}^{3}$ and $\rho_{\text {solidgrains }}=2.675 \mathrm{~g} / \mathrm{cm}^{3}$, initial total porosity equals 0.407 , and final porosity 0.455 . It must be emphasized that no other 
parameter than porosity controls the asymptotic water intake. The present final value is consequently really representative of the total amount of water entered into the sample (excluding the estimated evaporated part). As quoted above in $\S 2.2$, this variation should come from changes in the spatial distribution of porosity.

Stiffnesses $\kappa_{s 0}$ and $\kappa_{0}$ were difficult to discriminate precisely during the sensitivity analysis, because they counter-balance each other in some way. As a consequence, their present values are in an acceptable range, and are highly non-unique. Notwithstanding that stiffnesses play fundamental roles in the hydro-mechanical process, oedometer confined imbibition tests seem inappropriate to quantify them precisely. They should be characterized separately, with different experimental protocols, as suggested by Alonso et al (op. cit.).

The system appeared extremely sensitive to small percent-variations of $\alpha_{\kappa s}$ and $\alpha_{\kappa}$, but moderately sensitive to $\lambda_{0}$.

Consolidation pressure $P_{0}$, one of the key parameters of the problem (Demers 2002) because of its property to allow or limit yielding, as depicted on Fig. 6, cannot be estimated a priori. Any value between one atmosphere (no consolidation) and $40 \mathrm{MPa}$ (pellets molding pressure) would be acceptable because of the heterogeneity of the material.

The compaction pressure is significantly lower than the pellets initial consolidation (about $40 \mathrm{MPa}$ ). Hence, it is reasonable to think that powder alone has endured yielding during the preparative compaction. Some marginal areas where isostatic pressure has locally exceeded pellets consolidation should be considered as exceptions. It is thus probable that only powder plastifies at the beginning of the imbibition. Overall consolidation pressure should otherwise be higher. The - rather low $-P_{0}$ value found here suggests that the high-density, highly over-consolidated, stiff pellets undergo, by mechanical percolation, most of the compaction stress applied during the preparation of the sample. This process implies high compaction of small amounts of powder along the preferential stress paths, protecting the rest of the powder from further compaction. This "virgin" powder will plastify later under humidification. Somehow, there must be some fraction of the material left sufficiently unconsolidated by the preparatory compaction, in order to allow yielding during the following imbibition. If a homogeneous medium had been used, the consolidation pressure should have been equal to its compaction pressure. The intermediate value of $P_{0}$, computed as if the mixture had been an equivalent homogeneous material, is therefore, like all other estimated parameters, an "up-scaled" value. 


\section{Representativity of the characterization}

The powder/pellet mixture is obviously an heterogeneous medium. In theory, several samples of same height may behave differently from oneanother, even if they were prepared by the same scrupulous protocol. The reason for this is the diversity of their internal arrangement. At some sufficient size, these differences should not show anymore because there would be enough heterogeneity units to counter- balance one-another. It ensues from this that a sample would produce the same response as any other one, provided their sizes are above this threshold. Samples below the threshold should thence not represent large-scale behavior of the same material.

In our case, the length of heterogeneity of the material is typically the pellet size: $1 \times 2.5 \mathrm{~cm}$. It is commonly assumed in solute transport that about ten times this length are necessary to treat the material as a homogeneous medium (Sanchez 1980 ; Lewandowska and Auriault 1998). Applying this estimation in a qualitative way to hydro-mechanical behavior suggests that samples would not behave as a same homogeneous medium under an approximate minimal length $17 \mathrm{~cm}$. In other words, our three samples, their heights ranging from 5 to $12 \mathrm{~cm}$, should not a priori elect as representative of the average behavior of a FoCa powder/pellet mixture. This is one of the surprising results of this study, that small samples could be representative of a same, asymptotic material. It was expected before the study, that the response of the increasingly high samples would show a trend in, for example, swelling pressure at saturation. This study proved it was not the case. There may be several explanations for this: (i) the analogy with solute transport is inadequate, because the hydro-mechanical coupled processes are too different from solute transport, (ii) the heterogeneity is in fact very weak, and we actually deal with quasi-homogeneous media, (iii) the measured variables (axial stress and water intake) are not sensitive to heterogeneity and, by nature (like mass fractions), average disparities nearly independently of the sampling size.

This latter explanation does not hold if one considers how the key parameter $P_{0}$ (consolidation pressure) found equal to $2.3 \mathrm{MPa}$, differs from the compaction pressure $9 \mathrm{MPa}$ it should have had if the material had been homogeneous, and how strongly swelling pressure depends on this parameter. The second explanation is, for the same reason, swept. The first explanation solely remains. Let us furthermore remind that time scale variations, which depend only on saturation permeability, has been excluded from this discussion. 


\section{Conclusion}

The numerical analysis of the imbibition of $1.6 \mathrm{~g} / \mathrm{cm}^{3}$ dense samples of FoCa powder/pellet mixture brings a qualitative insight, in the light of the $\mathrm{BBM}$ model, to the interpretation of swelling pressure and water intake evolutions during an oedometer test. The swelling pressure variations - specially its temporary decrease - have been explained by yielding under stress increase, as an indirect consequence of radial confinement. This explanation may depend upon the chosen physical model though. For instance, our description may have been different if plasticity occurrence under suction decrease had been taken into account. Yielding would still have been incriminated however. In the case of a metric seal placed into a cylindrical access shaft, the boundary conditions should be strongly different. Hydration would occur by the side rather than from the lower base. Weaker confinement would be offered by the wall of the shaft and concrete packings. The yielding of the seal should be moderated by the higher connectivity of circular imbibition in comparison to mono-axial, and by host rock and concrete deformations. These weaker solicitations can possibly prevent the temporary decrease of the swelling pressure observed on the oedometer tests.

The general shape of the hydraulic and mechanical response of the samples has been satisfactorily reproduced by the model. A voluntarily limited set of parameters, determined by a rough sensibility analysis as well as arbitrary choices, has been calibrated. As usual in such a complex model, the calibration, which was performed by hand, is non unique. It gives nevertheless orders of magnitude of key parameters, like those related to imbibition dynamics. Stiffnesses and consolidation pressure proved to play major roles in the intensity and in the nature of the physical response of the samples. Several protocols should be conducted in order to really characterize the material, with proper discrimination between parameters, in order to reduce the equifinality of their values. More generally, left-out important processes like crystalline and osmotic swelling must be eventually incorporated in both hydraulic and mechanical models; for example by using the Barcelona Extended Model (Alonso et al. 1999). This is necessary to quantify how irreversible osmotic deformation would superimpose to stress-induced yielding, and maybe explain also the partial misfit of the swelling pressure simulation. On the other hand, the assumptions made in the hydraulic model showed acceptable a posteriori from the restrictive point of view of the water intake.

Statistical asymptotic stationarity was achieved with smaller samples that could have been inferred from stochastic theory applied to solute transport. 
Maybe because of the coupling of hydraulics to highly non-linear mechanical processes. Caution should be the rule in extending the results of this study to large scale seals in accordance to what has been said above, and also because: (i) higher scale heterogeneities come into place; and (ii) the calibration only apply to similar 1D situations with hydration from one side and perfect lateral confinement. Those restrictions would normally not be satisfied in actual sealing implementation.

From the industrial point of view, the principle of using a mixture of powder and pellets to make a seal proved interesting. Easy to built up, this material shows good swelling properties as well as a very low permeability. From our characterization, it appears that the mixture benefits from both components. First, it inherits from the powder its low effective consolidation pressure, that makes it malleable and would help in settling definitive high-precision cavity filling. Second, it inherits from the pellets their high stiffnesses, giving the whole an elastic reserve in case of future evolution of the cavity (widening by mechanical relaxation, fracturation, and so on). It should therefore profit of further studies and use.

\section{Acknowledgments}

This study is integrated information from the RESEAL I and RESEAL II projects. RESEAL I was a project co-funded by the European Commission and performed as part of the fourth EURATOM framework programme, key action Nuclear Fission safety (1994-1998), EC contract number FI4W-CT960025. RESEAL I was also co-funded by NIRAS-ONDRAF (coordinating the National Belgian Waste Management Program), ANDRA (the French National Radioactive Waste Management Agency) and ENRESA (the Spanish National Radioactive Waste Management Agency). The RESEAL II project is co-funded by the European Commission and performed as part of the fifth EURATOM framework programme, key action Nuclear Fission (1998-2002), EC contract number FIKW-CT2000-00010. The project is also co-funded by NIRAS-ONDRAF and ANDRA.

\section{A List of symbols}

Dimensions are written between square brackets.

Hydraulic variables:

- $S$ : water saturation [-], 
- $\omega$ : cinematic as well as total porosity [-],

$-\vec{U}_{l}$ : Darcy's velocity of the liquid phase $[\mathrm{m} / \mathrm{s}]$,

- $q_{w}:$ water source/sink term $\left[s^{-1}\right]$,

- $s$ : suction $=P_{g}-P_{l}$ (positive in non saturated, null otherwise) $[P a]$,

- $P_{g}:$ gas pressure $=$ constant $[P a]$,

- $P_{l}$ : liquid water pressure $[P a]$,

- $K_{l}$ : permeability to water $[\mathrm{m} / \mathrm{s}]$,

$-k_{l}^{r}$ : relative permeability to water [-],

$-k_{\text {int }}:$ intrinsic permeability $\left[\mathrm{m}^{2}\right]$,

- $\mu_{w}$ : water dynamic viscosity $[\mathrm{kg} / \mathrm{m} / \mathrm{s}]$,

- $\omega_{k}$ : reference Kozeny porosity [-],

$-k_{\text {int }}^{0}$ : reference Kozeny intrinsic permeability $\left[\mathrm{m}^{2}\right]$,

Mechanical variables:

- $\overline{\bar{\sigma}}$ : total stress tensor, positive on compression $[P a]$; subscript $d$ goes for the deviatoric part,

$-\vec{f}$ : volumetric forces $\left[N / m^{3}\right]$,

- $\bar{\varepsilon}$ : strain tensor, positive on dilatation [-]; subscripts $\sigma$ or $s$ mean strain under stress or suction variations respectively, while superscripts $\nu$ goes for volumetric strain, and superscripts $e$ or $p$ specify the elastic or plastic part of the deformation respectively; as with stress, subscript $d$ goes for the deviatoric part,

- $\overline{\bar{I}}$ : identity tensor $[-]$,

$-\overline{\overline{\sigma^{\prime}}}$ : modified stress $[P a]$,

- $p^{\prime}:$ isostatic modified pressure $=\frac{1}{3} \operatorname{tr} \overline{\overline{\sigma^{\prime}}}[P a]$,

- $\overline{\bar{\sigma}}_{d}:$ deviatoric stress $=\overline{\overline{\sigma^{\prime}}}-p^{\prime} \overline{\bar{I}}[P a]$

- $q$ : confinement stress $=\sqrt{\frac{3}{2}} \sqrt{\overline{\bar{\sigma}}_{d}:{ }^{t} \overline{\bar{\sigma}}_{d}}[P a]$,

- $M$ : slope of the critical state line [-],

- $P_{s}$ : cohesion pressure $[P a]$,

- $P_{0}$ : consolidation pressure $[P a]$,

- $P_{0}^{*}$ : consolidation pressure for saturated conditions $[P a]$,

- $s_{0}$ : suction limit $[\mathrm{Pa}]$,

$-\mathcal{F}$ : Loading/Collapse elastic function $\left[\mathrm{Pa}^{2}\right]$,

$-\mathcal{F}^{\prime}$ : Suction Increase elastic function $\left[\mathrm{Pa}^{2}\right]$,

$-\mathcal{G}$ : plastic potential $\left[\mathrm{Pa}^{2}\right]$

$-e:$ void ratio $=\omega /(1-\omega)[-]$,

$-k_{s}$ : parameter describing the increase of cohesion with suction

$-\kappa$ : elastic stiffness to $p^{\prime}$ increase [-],

$-\kappa_{0}$ : its value at saturation $[-]$, 
- $\kappa_{s}$ : elastic stiffness to suction increase [-],

$-\kappa_{s 0}$ : its value at saturation and at the reference isostatic pressure $p_{\text {ref }}[-]$,

- $P_{\text {atm }}:$ atmospheric pressure $[P a]$,

- $\lambda$ : stiffness to $p^{\prime}$ increase for virgin states of the material [-],

- $\lambda_{0}$ : its value at saturation [-],

$-\lambda_{s}$ : stiffness to suction increase for virgin states of the material $[-]$,

- $G$ : shear modulus $[P a]$,

\section{B Appendix : Physical model}

The hydraulic and mechanical equations are presented hereafter, along with the corresponding physical laws. Tensors are written in bold characters. The a priori value of the parameters is also given, but some of them were changed afterward during the parameter estimation on the basis of the experimental results.

\section{B.1 Hydraulic equation and properties}

\section{Water conservation:}

The Richard's equation seemed sufficient to describe the hydraulic phenomena, because the imbibition kinetics in clay are slow enough to consider immediate equilibrium of gas pressure with ambient air. Conservation of (incompressible) water volume thus reduces to:

$$
\frac{\partial S \omega}{\partial t}+\operatorname{div} \vec{U}_{l}=q_{w}
$$

\section{Physical laws and parameters:}

A Van Genuchten model described the capillary law:

$$
s=s_{G}\left[S^{-1 / \lambda_{G}}-1\right]^{1-\lambda_{G}}
$$

Darcy's law for water transfer was assumed:

$$
\vec{U}_{l}=-K_{l} \vec{\nabla} P_{l}
$$

with

$$
K_{l}=k_{l}^{r} k_{i n t} / \mu_{w}
$$


Kozeny-Carman expression for variations of intrinsic permeability with porosity applied:

$$
k_{i n t}=k_{i n t}^{0} \frac{\omega^{3}}{(1-\omega)^{2}} \frac{\left(1-\omega_{k}\right)^{2}}{\omega_{k}^{3}}
$$

while a simple power law was used for relative permeability:

$$
k_{l}^{r}=A S^{B}
$$

The nominal parameter values were the following: $\lambda_{G}=0.27, s_{G}=17 \mathrm{MPa}$, $\mu_{w}=0.89 \cdot 10^{3} \mathrm{~kg} / \mathrm{m} / \mathrm{s}$ at $25 \mathrm{C}, k_{\text {int }}^{0}=3.4 \cdot 10^{-21} \mathrm{~m}^{2}, \omega=\omega_{k}=0.407, A=1$, $B=3$.

\section{B.2 Mechanical equation and properties}

\section{Mechanical equilibrium:}

The mechanical equilibrium of internal forces has been assumed throughout the imbibition, because forces equilibrate very quickly one-another in comparison with the hydraulic kinetics, and the transformation can be assumed quasi-static from the mechanistic point of view:

$$
\operatorname{div} \overline{\bar{\sigma}}=\vec{f}
$$

\section{Physical laws and parameters:}

The BBM model (Alonso et al. 1990), enhanced by Gens and Olivella (Gens, A., Olivella, S. 2000. Expertise de la problématique THM-Gaz dans le cadre de l'étude de la faisabilité d'un stockage en profondeur, internal report ANDRA, C RP 0UPC 00-001), was used to describe the mechanical behavior of the swelling clay material, where two independent variables control deformations. These two variables are suction $s$ and modified stress $\overline{\overline{\sigma^{\prime}}}$. The latter being equal to net stress $\overline{\bar{\sigma}}_{\text {net }}=\sigma-P_{g} \overline{\bar{I}}$ under unsaturated conditions, and to effective stress $\overline{\bar{\sigma}}_{\text {eff }}=\sigma-P_{l} \overline{\bar{I}}$ otherwise.

The elastic domain is limited by two conditions: (i) the Loading/Collapse curve for $p^{\prime}$ increase

$$
\begin{gathered}
\mathcal{F}=3 \overline{\bar{\sigma}}_{d}^{2}-M^{2}\left(p^{\prime}+P_{s}\right)\left(P_{0}-p^{\prime}\right) \leq 0 \\
P_{0}(s)=P^{c}\left(\frac{P_{0}^{*}}{P^{c}}\right)^{\frac{\lambda_{0}-\kappa_{0}}{\lambda(s)-\kappa(s)}} \\
P_{s}=-k_{s} s
\end{gathered}
$$


and (ii) the Suction Increase curve for $s$ increase:

$$
\mathcal{F}^{\prime}=s-s_{0} \leq 0
$$

Elastic and plastic deformations are defined separately in two steps by estimating on the one hand their volumetric part, and on the second hand their deviatoric part:

$$
\begin{aligned}
\delta \varepsilon_{\sigma}^{\nu, e l} & =-\frac{\kappa}{1+e} \frac{\mathrm{d} p^{\prime}}{p^{\prime}} \\
\delta \varepsilon_{s}^{\nu, e l} & =-\frac{\kappa_{s}}{1+e} \frac{\mathrm{d} s}{s+P_{a t m}}
\end{aligned}
$$

Linear elasticity apply to deviatoric stress-induced strain:

$$
\mathrm{d} \overline{\bar{\varepsilon}}_{d, \sigma}^{e l}=-\frac{1}{2 G} \mathrm{~d} \overline{\bar{\sigma}}_{d}
$$

while swelling is assumed isotropic:

$$
\mathrm{d} \overline{\bar{\varepsilon}}_{d, s}^{e l}=\overline{\overline{0}}
$$

Plastic deformations have these properties to be symmetric (no deviator) and that Loading/Collapse consolidation reciprocally generate Suction Increase consolidation. Namely: $\delta \overline{\bar{\varepsilon}}_{d}^{p l}=0$ and $\delta \varepsilon_{\sigma}^{\nu, p l}=\delta \varepsilon_{s}^{\nu, p l}=\delta \varepsilon^{p l}$. Plastic strains are deduced from the non-standard plastic potential

$$
\mathcal{G}=3 \gamma \overline{\bar{\sigma}}_{d}^{2}-M^{2}\left(p^{\prime}+P_{s}\right)\left(P_{0}-p^{\prime}\right)+\left(s-s_{0}\right)
$$

where

$$
\gamma=\frac{M(9-M)(3-M)}{9(6-M)} \frac{\lambda_{0}}{\lambda_{0}-\kappa_{0}}
$$

while variations of plastic limits are given by the following laws:

$$
\delta \varepsilon_{\sigma}^{\nu, p l}=-\frac{\lambda_{0}-\kappa_{0}}{1+e} \mathrm{~d} \ln P_{0}^{*}
$$

$P_{0}$ being equal to $p^{\prime}$ if reaching Loading/Collapse curve. And

$$
\delta \varepsilon_{s}^{\nu, p l}=-\frac{\lambda_{s}-\kappa_{s}}{1+e} \mathrm{~d} \ln \left(s_{0}+P_{a t m}\right)
$$

$s_{0}$ being equal to $s$ if reaching Suction Increase curve. 
The following laws applied to stiffness variations:

$$
\begin{aligned}
\kappa(s) & =\min \left[\kappa_{0} ; \kappa_{0}\left(1-\alpha_{\kappa} \ln \left(10^{-6} s\right)\right)\right] \\
\kappa_{s}\left(s, p^{\prime}\right) & =\kappa_{s 0}\left(1-\alpha_{\kappa_{s}} \ln \left(p^{\prime} / p_{r e f}\right)\right) e^{-\beta_{\kappa_{s}} s}
\end{aligned}
$$

The shear modulus is deduced from a linear elastic model with constant Poisson's coefficient:

$$
G=\frac{3(1-2 \nu)}{2(1+\nu)} \frac{(1+e) p^{\prime}}{\kappa(s)}
$$

Plastic deformation is quantified by a constant $\lambda_{s}$ for suction increase, and a variable $\lambda(s)$ for Loading/Collapse:

$$
\lambda(s)=\lambda_{0}\left[(1-r) e^{-\beta_{\lambda} s}+r\right]
$$

The nominal parameter values, given here for orders of magnitude, are $M=1.5, P^{c}=1 \mathrm{~atm}, k_{s}=0.1, \kappa_{0}=0.015, \alpha_{\kappa}=0.12, \kappa_{s 0}=0.45, \alpha_{\kappa_{s}}=$

$0.1638, p_{\text {ref }}=10^{4} \mathrm{~Pa}, \beta_{\kappa_{s}}=1.1 \cdot 10^{-8} \mathrm{~Pa}^{-1}, \lambda_{0}=0.22, r=0.75, \beta_{\lambda}=0.05$ $M P a^{-1}, \nu=0.4$.

\section{References}

[AEN 1999] AEN. 1999. Confidence in the Long-term Safety of Deep Geological Repositories Its Development and Communication, OCDE/AEN, Paris, France.

[Achari et al. 1999] Achari, G. Joshi, R.C., Bentley, L.R., Chatterji, S. 1999. Prediction of the hydraulic conductivity of clays using the electric double layer theory, in Can. Geotech. J., 36, pp 783-792.

[Alonso et al. 1990] Alonso, E.E., Gens, A., Josa, A. 1990. A constitutive model for partially saturated soils, in Géotechnique, 40(310), pp 405430 .

[Alonso et al. 1999] Alonso, E.E., Vaunat, J., Gens, A. 1999. Modelling the mechanical behaviour of expansive clays, in Engineering Geology, 54, pp 173-183.

[Barnel 2004] Barnel, N. 2004. Comportement en température des argiles de $\mathrm{BO}$ : réexamen de la phénoménologie et amélioration des modèles existants, Ph.D. Thesis, Université d'Evry, France. 
[Carman 1937] Carman, P. 1937, Fluid flow through a granular bed, in Trans. Inst. Chem. Eng., 15, pp 150-167.

[Chapuis and Aubertin 2003] Chapuis, R.P., M. Aubertin. 2003. On the use of the Kozeny/Carman equation to predict the hydraulic conductivity of soils, Can. J. Geotech./Rev. Can. Geotech., 40(3), pp 616-628

[Chateau and Dormieux 1998] Chateau, X., Dormieux, L. 1998. Approche micromécanique du comportement d'un milieu poreux non saturé, in C.R. Acad. Sci. Paris, 326, series II b, pp 533-538.

[Coussy 2004] Coussy, O. 2004. Poromechanics, 320p, ed. John Wiley and Sons, ISBN 0-470-84920-7.

[Crawford et al. 2002] Crawford, B.R., Myers, R.D., Woronow, A., Faulkner, D.R., Rutter, E.H. 2002. Porosity-permeability relationships in claybearing fault gouge, in Proc. of SPE/IRSM Rock Mechanics Conf., Irving, Texas, 20-03 Oct. 2002.

[de Bruyn and Boyazis 1996] de Bruyn, D., Boyazis, J.P. 1996. The HADES Underground Research Facility for the Geological Disposal of High-Level Radioactive Waste and its future extension within the PRACLAY program, Proc. ENS Class 1 Topical Meeting on Research Facilities for the Future of Nuclear Energy, Brussels, Belgium, June 1996, ed. H. At Abderrahim, World Scientific, pp 479-485.

[Dormieux et al. 2003] Dormieux, L., Lemarchand, E., Coussy, O. 2003. Macroscopic and micromechanical approaches to the modeling of the osmotic swelling in clays, in Transport In Porous Media, 51(1-2).

[de Marsily 2002] de Marsily, G., Goncalves, J., Violette, S., Castro, M.C. 2002. Migration mechanisms of radionuclides from a clay repository toward adjacent aquifers and the surface. in Applied Physics, 3, pp 945-959.

[Demers 2002] Demers, D., Leroueil, S. 2002. Evaluation of preconsolidation pressure and the overconsolidation ratio from piezocone tests of clay deposits in Quebec, in Can. J. Geotech./Rev. Can. Geotech., 39(1), pp 174-192.

[Dixon et al. 1999] Dixon, D.A., Graham, J., Gray, M.N. 1999. Hydraulic conductivity of clays in confined tests under low hydraulic gradients, in Can. J. Geotech./Rev. Can. Geotech., 33(5), pp 815-825. 
[Dudoignon et al. 2004] Dudoignon, P., Gélard, D., Sammartino, S. 2004. Cam-Clay and hydraulic conductivity diagram relations in consolidated and sheared clay-matrices, in Clay Minerals, 39(3), pp 267-279.

[Gens and Alonso 1992] Gens, A., Alonso, E.E. 1992 A framework for the behaviour of unsaturated expansive clays, in Can. J. Geotech./Rev. Can. Geotech., 29, pp 1013-1032.

[Genty et al. 1999] Genty, A., Le Potier, C., Renard, P. 1999. Upscaling of heterogeneous relative permeability curves, in Annual Conf. of the Int. Assoc. for Math. Geol., IAMG'99 proceedings, Trondheim, Norway.

[Imbert et al. 2002] Imbert, C., Gatabin, C., Maugis, P., Leboulch, D., Mouche, E. 2002. Hydro mechanical behaviour of a heterogeneous swelling clay material in Poromechanics II, Proc. of the second Biot Conference on poromechanics, Grenoble, France, Balkema Publishers.

[Jeffries 1992] Jeffries, R.M. 1992. Interclay II Project a Coordinated Bench Mark Exercise on the Rheology of Clays, European Commission, ed. Softcover, ISBN: 92-826-5904-6 / 9282659046

[Jullien et al. 2005] Jullien, M., Raynal, J., Kohler, É., Bildstein, O. 2005. Physicochemical reactivity in Clay-Rich Materials: Tools for safety assessment, in Can. J. Geotech./Rev. Can. Geotech., 29, pp 1013-1032.

[Kozeny 1927] Kozeny, J. 1927, Über kapillare Leitung der Wasser in Boden, in Sitzungsber. Akad. Wiss. Wien, 136, pp 271-306.

[Le Potier et al. 1998] Le Potier, C., Genty, A., Millard, A., Mouche, E., Cany, F., Benet, L.V., Plas, F., Palut, J.M. 1998. Modeling of the Thermo-Hydro-Mechanical behaviour of clay materials with Castem2000, Proceedings of the 5th International Workshop on Key Issues in Waste Isolation Research, held in Barcelona.

[Lewandowska and Auriault 1998] Lewandowska, J., Auriault, J.-L. 1998. Scale separation in diffusion/dispersion tests in porous media, in Poromechanics - A tribute to Maurice A. Biot, Proc. of the Biot Conference on poromechanics, Louvain-la-Neuve, Belgique, Balkema Publishers.

[Ontoy et al. 2003] Ontoy, Y., Molling, P.L., Xu, T., Spycher, N., Parini, M., Pruess, K. 2003. Scaling of hot brine injection wells: supplementing field studies with reactive transport modeling., in Proc. of TOUGH Symposium 2003, Lawrence Berkeley National Laboratory, Berkeley, California, May 12-14. 
[Pasquiou 2001] Pasquiou, A. 2001. Pellets d'argiles gonflantes. Elaboration et caractérisation hydromécanique, Thesis, Université des Sciences et Techniques de Lilles, Lilles, France.

[Robinet et al. 1995] Robinet, J.C., Rahboui, A., Plas, F., Lebon, P. 1995. A constitutive thermomechanical model for saturated clays, Journal Engineering Geology, 145-169.

[Robinet et al. 2002] Robinet, J.C., Ducoulombier, A., Pothier, C. , Jullien, A., Plas, F. 2002. Evaluation of a constitutive model for expansive clays, Engineering geology, Elsevier ed., 41, pp 145-169.

[Rubin 1990] Rubin, Y. 1990. Stochastic modeling of macrodispersion in heterogeneous porous media, in Water Resources Research, 26(1), pp 133141.

[Sanchez 1980] Sanchez Palencia E. 1980. Non homogeneous media and vibration theory, Lecture notes in physics, Springer Verlag.

[Saiyouri et al. 2004] Saiyouri, N., Tessier, D., Hicher, P.Y. 2004. Experimental study of swelling in unsaturated compacted clays, in Clay Minerals, 39(4), pp 469-479.

[Scheidegger 1960] Scheidegger, A.E. 1960. The Physics of Flow through Porous Media, 313 pp., University of Toronto Press.

[Suzuki 2005] Suzuki, S. Prayongphan, S., Ichikawa, Y., Chae, B.G. 2005. In situ observations of the swelling of bentonite aggregates in $\mathrm{NaCl}$ solution, in Applied Clay Science, 31, pp 96-109.

[Villar and Martin 1996] Villar, M.V., Martin, P.L. 1996. Suction controlled oedometer tests in montmorillonite clay. Preliminary results, in Engineering geology of waste disposal, Geological Society Engineering Geology special publication $n^{\circ} 11$, ed. S.P. Bentley, The Geological Society, London, 309-311.

[Villar 2006] Villar, M.V. 2006. Infiltration tests on a granite/bentonite mixture: Influence of water salinity, in Applied Clay Science, 31, pp 96-109.

[Volckaert et al. 1996a] Volckaert, G., Bernier, F., Alonso, E., Gens, A., Samper, J., Villar, M.V., Martin-Martin, P.L., Cuevas, J., Campos, R., Thomas, H., Imbert, C., Zingarelli, V. 1996. Thermal-hydraulicmechanical and geochemical behavior of the clay barrier in radioactive waste repositories (model development and validation) - Final Report, 
Luxembourg: Office for Official Publications of the European Communities, Nuclear Science and Technology series, EUR 16744 EN, ISBN 92827-5743-9.

[Volckaert et al. 1996b] Volckaert, G., Bernier, F., Dardaine, M. 1996. Demonstration of the In Situ Application of an Industrial Clay-Based Backfill Material (BACCHUS 2), Luxembourg: Office for Official Publications of the European Communities, Nuclear Science and Technology series, EC contract FI2W-CT91-0098, EUR 16860 EN.

[Volckaert et al. 2000] Volckaert, G., Dereeper B., Put M., Ortiz L., Gens A., Vaunat J., Villar M.V., Martin P.L., Imbert C., Lassabatère T., Mouche E., Cany F. 2000. A large-scale in situ demonstration test for repository sealing in an argillaceous host rock. Reseal Project - Phase I, Luxembourg: Office for Official Publications of the European Communities, Nuclear Science and Technology series, EUR 19612 EN.

[Wong 2003] Wong, R.C.K. 2003. A model for strain-induced permeability anisotropy in deformable granular media, in Can. Geotech. J., 40, pp 95-106.

[Yong 1999] Yong, R.N. 1999. Soil suction and soil-water potentials in swelling clays in engineered clay barriers, in Engineering Geology, 54, pp 3-13. 


\section{Figure Captions}

Figure 1

Schematic presentation of the experimental device. In some cases, water is introduced under pressure by a pump.

Figure 2

Experimental results for three sample heights: 5, 10 and $12 \mathrm{~cm}$. Time $t$ has been normalized with the first maximum occurrence time $t_{1}$. The bigger sample $(12 \mathrm{~cm})$ has been imbibed with 6 Bar water pressure instead of 0.1 Bar for the smaller ones.

(a) Normalized water intake at the bottom of the samples (excluding evaporation). This ratio should reach porosity after saturation. (b) Swelling pressure $P_{g}$ at the top of the samples.

Figure 3

Experimental swelling pressure of an homogeneous sample made of powder only, $5 \mathrm{~cm}$ high, density $1.42 \mathrm{~g} / \mathrm{cm}^{3}$. Notice the weaker swelling pressure and the lower asymptote in comparison with powder/pellets mixtures.

Figure 4

Temporal evolution at different vertical positions, for an initial consolidation pressure $P_{0}=2.3 \mathrm{MPa}$. It shows how plastic deformation $\varepsilon_{r}^{p l, \sigma}$ begins when radial stress $\sigma_{r r}$ exceeds $P_{0}$. Black squares indicate initial point.

Figure 5

Spatial evolution at different times, up to 100 days. $z$ is the vertical upward axis. The arrows indicate the direction of increasing times $(0,0.5 \mathrm{~h}, 1 \mathrm{~h}$, 2h, 6h, 1d, 2d, 3d, 4d, 5d, 6d, 7d, 8d, 16d ,31d, 46d, 61d, 76d and 90d). Letters $\mathrm{I}$ and $\mathrm{F}$ denote the initial and final times respectively.

Figure 6

Influence of consolidation pressure $P_{0}$ on swelling pressure $P_{g}$ for a synthetic arbitrary sample.

Figure 7

Experimental and computed calibrated Swelling Pressure and Water Intake for the $5 \mathrm{~cm}$ high sample. 


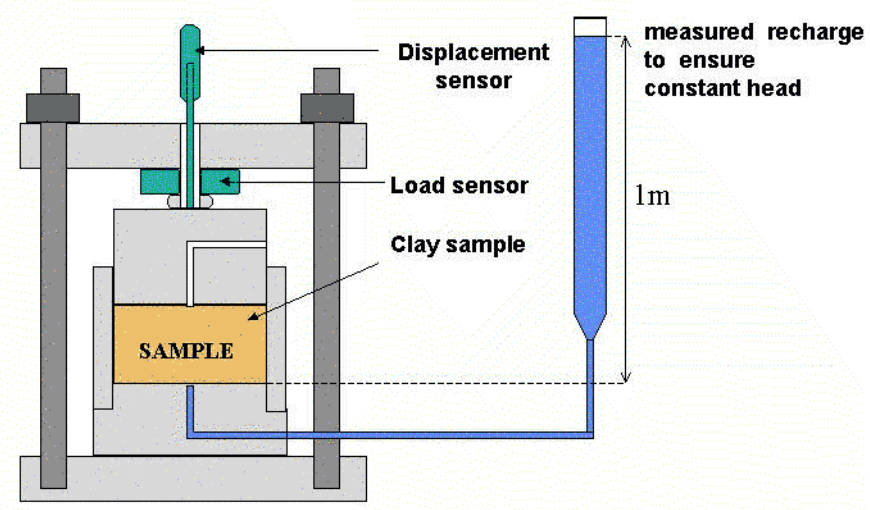

Figure 1: Schematic presentation of the experimental device. In some cases, water is introduced under pressure by a pump. 

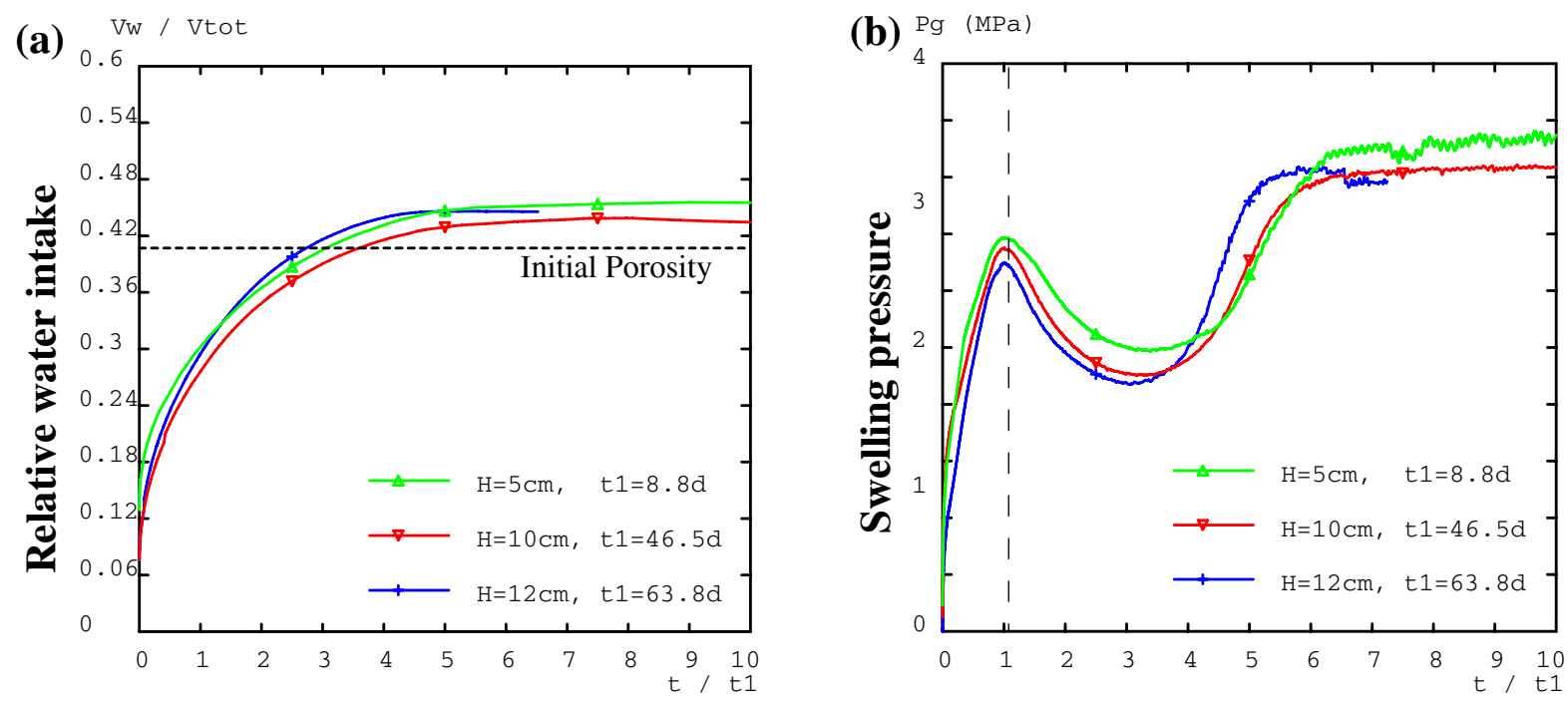

Figure 2: Experimental results for three sample heights: 5, 10 and $12 \mathrm{~cm}$. Time $t$ has been normalized with the first maximum occurrence time $t_{1}$. The bigger sample $(12 \mathrm{~cm})$ has been imbibed with 6 Bar water pressure instead of 0.1 Bar for the smaller ones.

(a) Normalized water intake at the bottom of the samples (excluding evaporation). This ratio should reach porosity after saturation.

(b) Swelling pressure $P_{g}$ at the top of the samples. 


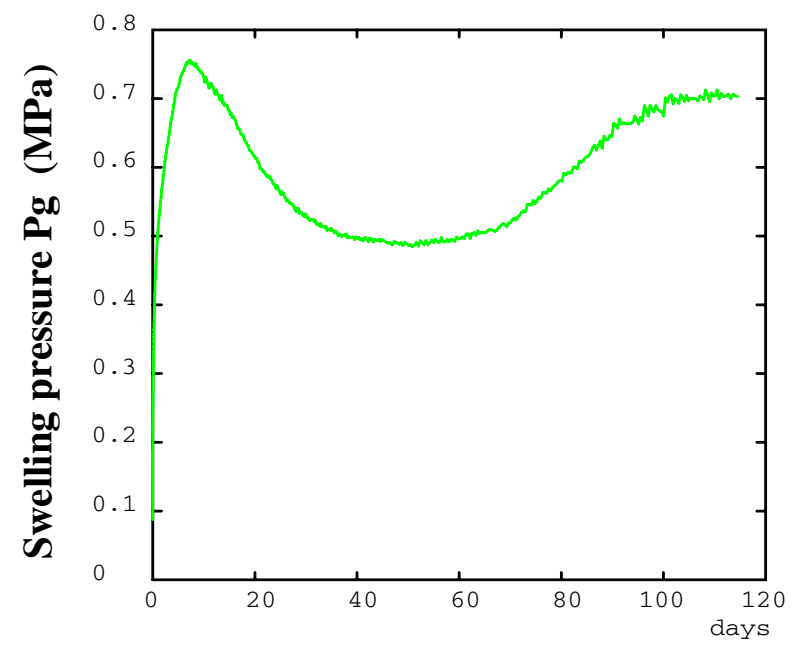

Figure 3: Experimental swelling pressure of an homogeneous sample made of powder only, $5 \mathrm{~cm}$ high, density $1.42 \mathrm{~g} / \mathrm{cm}^{3}$. Notice the weaker swelling pressure and the lower asymptote in comparison with powder/pellets mixtures. 

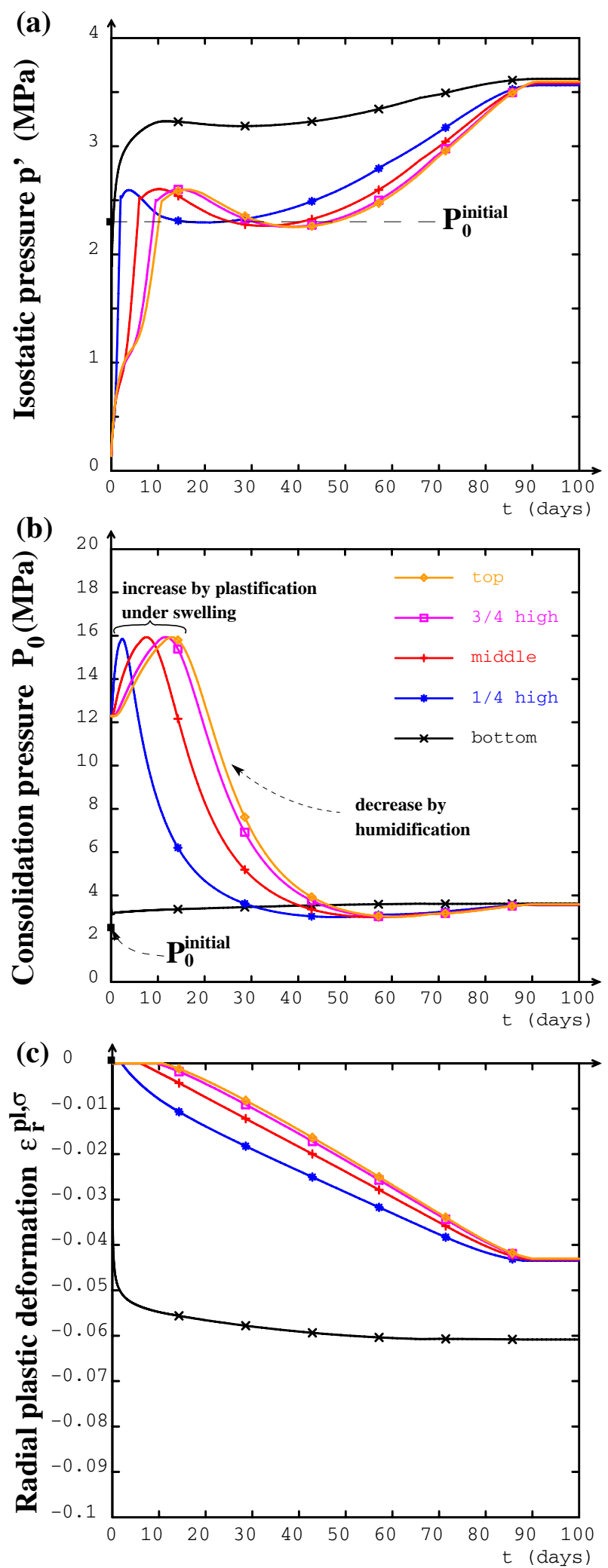

Figure 4: Temporal evolution at diffi:sent vertical positions, for an initial consolidation pressure $P_{0}=2.3 \mathrm{MPa}$. It shows how plastic deformation $\varepsilon_{r}^{p l, \sigma}$ begins when radial stress $\sigma_{r r}$ exceeds $P_{0}$. Black squares indicate initial point. 

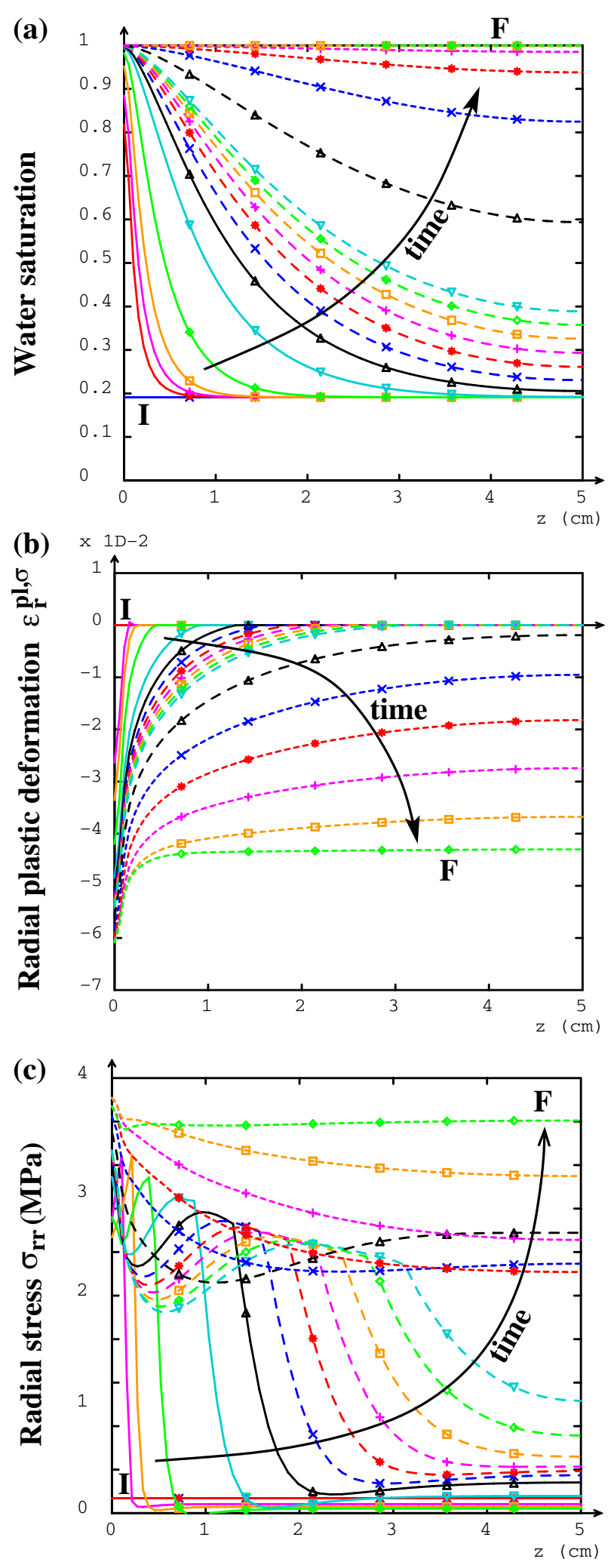

Figure 5: Spatial evolution at different times, up to 100 days. $z$ is the vertical upward axis. The arrows indicate the direction of increasing times (0, 0.5h, 1h, 2h, 6h, 1d, 2d, 3d, 4d, 5d, 6d, 7d, 8d, 16d ,31d, 46d, 61d, 76d and 90d). Letters I and F denote the initial and final times respectively. 


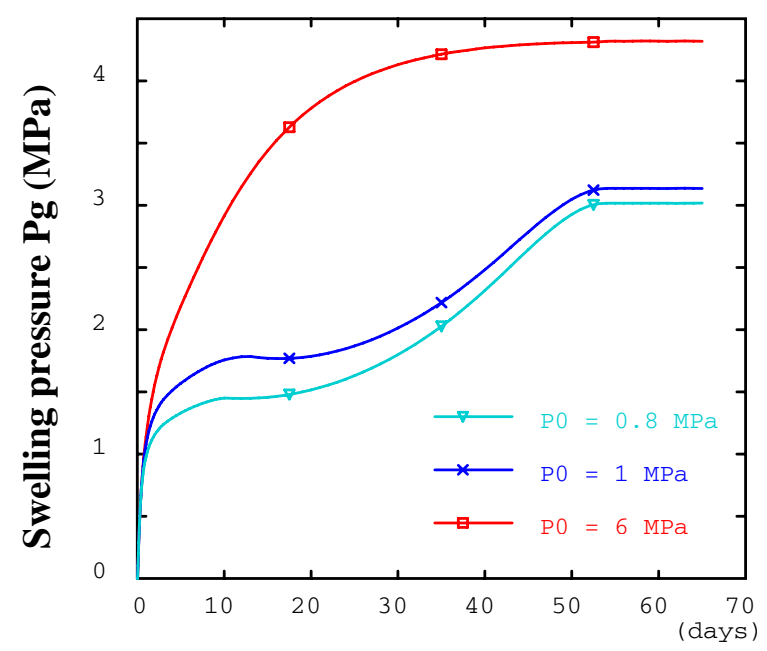

Figure 6: Influence of consolidation pressure $P_{0}$ on swelling pressure $P_{g}$ for a synthetic arbitrary sample. 


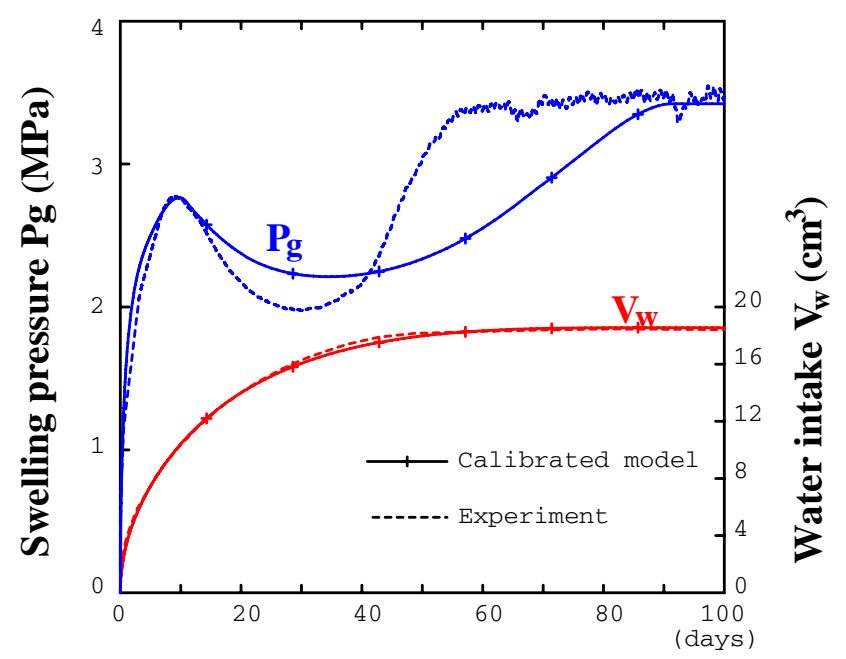

Figure 7: Experimental and computed calibrated Swelling Pressure and Water Intake for the $5 \mathrm{~cm}$ high sample. 